\title{
Eficácia e fitotoxicidade de herbicidas aplicados para o manejo de plantas daninhas em cevada ${ }^{1}$
}

\section{Chemical management alternatives weed infests barley crop}

Leandro Galon ${ }^{2}$; César Tiago Forte ${ }^{3}$; Renato Kujawiski ${ }^{4}$; André Luiz Radunz ${ }^{5}$; Felipe Adelio de David $^{3}$; Gismael Francisco Perin ${ }^{2}$; Camile Thais Castoldi ${ }^{3}$; Lauri Lourenço Radunz ${ }^{2}$

Resumo - O azevém é uma das principais plantas daninhas que infesta a cultura da cevada, ocasionando elevadas perdas de produtividade e da qualidade dos grãos dessa cultura. Diante disso, objetivou-se com o trabalho, identificar possíveis alternativas para o manejo químico de azevém infestante da cevada, bem como verificar a fitotoxicidade de herbicidas sobre os componentes de rendimento da cultura. Para tanto foi instalado um experimento, a campo, em blocos casualizados, arranjados em esquema fatorial 2 x 12, com quatro repetições. $\mathrm{O}$ fator A foi constituído pelos genótipos de cevada (MN610 e Crioula) e o B pelos herbicidas (iodosulfuron; imazethapyr + imazapic; clomazone; propanil; oxyfluorfen; metsulfuron-methyl; 2,4-D; cyhalofop-p-butil; penoxsulam e pyroxsulam), mais duas testemunhas uma capinada e outra sem capina. $\mathrm{O}$ genótipo MN610 apresentou elevada sensibilidade ao clomazone e ao imazethapyr + imazapic, tendo seus componentes de rendimento de grãos afetados negativamente na presença desses produtos. Já o genótipo Crioula, apresentou-se mais tolerante ao iodosulfuron, propanil, pyroxsulam, imazethapyr + imazapic, clomazone e oxyfluorfen, com menor redução nos componentes do rendimento da cevada. Os herbicidas aplicados podem, dependendo do genótipo, reduzir os componentes de rendimento da cultura, o que consequentemente afetará a produtividade de grãos da cevada. $\mathrm{O}$ iodosulfuron apresentou a melhor resposta para o controle de azevém, mantendo a cevada livre de planta daninha, aliado a uma menor fitotoxicidade a cultura.

Palavras-chaves: Hordeum vulgare, Lolium multiflorum, manejo químico

\begin{abstract}
Ryegrass is the main weed that infests barley, causing high yield losses and quality of grain. Thus, this work intended to evaluate the selectivity and efficacy of herbicides for weed control in barley. For such, we carried out an experiment in the field with randomized block design in factorial scheme $2 \times 12$, with four replications. Factor A is composed by the barley genotypes MN610 and Criola and factor B by herbicides: iodosulfuron imazethapyr + imazapic; clomazone; propanil; oxyfluorfen; metsulfuron-methyl; 2,4-D; cyhalofop; penoxsulam; were allocated pyroxsulam, weeded and infested. MN610 genotype showed high sensitivity to clomazone and imazethapyr + imazapic, with its yield components reduced in the presence of these products. Criola genotype presented itself with more tolerance to iodosulfuron, propanil, pyroxsulam,

\footnotetext{
${ }^{1}$ Recebido para publicação em 30/07/2014 e aceito em 31/05/2015.

2 Professor da Universidade Federal da Fronteira Sul (UFFS), Câmpus Erechim. Erechim, RS, BR. Email: leandro.galon@uffs.edu.br. (*autor para correspondência).

${ }^{3}$ Mestrando do Programa de Pós-Graduação em Ciência e Tecnologia Ambiental da Universidade Federal da Fronteira Sul (UFFS), Câmpus Erechim. Erechim, RS, BR.

${ }^{4}$ Acadêmico de Agronomia da Universidade Federal da Fronteira Sul (UFFS), Câmpus Erechim. Erechim, RS, BR.

${ }^{5}$ Bolsista DTI/FAPERGS/CAPES/UFFS, Câmpus Erechim/RS. Erechim, RS, BR.
} 
imazethapyr + imazapic, clomazone and oxyfluorfen because its yield components were lesser negatively affected by herbicides. The herbicides may cause, depending on the genotype, reduction in yield components of culture, which consequently affect the grain yield of barley. The herbicide that had the best response was iodosulfuron, keeping the crop free of weeds and combined with a lower phytotoxicity culture. The herbicide that had the best response was iodosulfuron, it kept the crop free of weeds with lowest phytotoxicity to culture.

Keywords: Hordeum vulgare, Lolium multiflorum, chemical management

\section{Introdução}

A cevada (Hordeum vulgare) é considerada uma espécie de ampla adaptabilidade ecológica, com uso na alimentação humana e animal, sendo matériaprima para a indústria cervejeira, devido à superioridade de seu malte. A cultura destaca-se entre as graníferas mais produzidas, ocupando a quinta posição em termos de importância econômica no mundo (FAO, 2015). A redução da produtividade de grãos, bem como da qualidade destes, principalmente para a produção de malte, podem estar associados ao manejo inadequado adotado para a cultura, especialmente no controle de plantas daninhas.

Devido a isto, o manejo das plantas daninhas é uma das práticas culturais que devem ser empregadas para a obtenção de maiores produtividades da cevada. As plantas daninhas competem com as culturas pelos recursos disponíveis no ambiente, liberaram substâncias alelopáticas ou podem hospedar pragas e doenças e, como consequência, ocasionarem perdas de produtividade e de qualidade dos grãos (Vargas et al., 2007; Galon et al., 2010; Galon et al., 2011).

Como método de controle para as plantas daninhas que infestam a cultura da cevada, em especial o azevém (Lolium multiflurum) e a nabiça (Raphanus sp.), são utilizados herbicidas. Principalmente em função da praticidade, eficiência e do menor custo quando comparados a outros métodos de controle (Christoffoleti et al., 2006).

$\mathrm{Na}$ atualidade são escassos os herbicidas registrados para o controle de plantas daninhas infestantes da cevada. Como exemplo tem-se o 2,4-D e o metsulfuron-methyl recomendados para o controle de plantas daninhas em pósemergência (Brasil, 2015). No entanto, os herbicidas podem exercer efeitos diretos e indiretos no crescimento e desenvolvimento das culturas cultivadas (Das et al., 2003; Rizzardi et al., 2003), tais como, alterações na absorção de nutrientes, sintomas de intoxicação e desregulação dos mecanismos de defesa da planta a determinados fatores estressantes, oxidação celular, dentre outros, que não são perceptíveis e nem amplamente considerados (Das et al., 2003; Rizzardi et al., 2003; Feng et al., 2005; Tuffi Santos et al., 2007; Galon et al., 2009). Além dos efeitos ocasionados pelos herbicidas citados anteriormente esses ainda podem ocasionar interferência negativa sobre os componentes do rendimento de grãos das culturas ou mesmo não apresentarem eficácia para o controle de plantas daninhas infestantes da cultura.

Da mesma forma, o genótipo pode influenciar nas respostas à aplicação dos diferentes herbicidas para controle das plantas daninhas. Pesquisas realizadas com outras culturas, como a cana-de-açúcar (Ferreira et al., 2005; Galon et al., 2009), o trigo (Paula et al., 2011), o arroz (Pinto et al., 2008) e a soja (Agostinetto et al., 2009), demonstram distintas respostas de genótipos à aplicação de herbicidas, tendo como consequências frequentes problemas de intoxicação e, às vezes, perda de produtividade das culturas ou mesmo interferência na qualidade do produto final.

Nesse sentido torna-se importante testar novas moléculas de herbicida mesmo que não sejam registrados para a cultura, para avaliar a eficácia de controle de plantas daninhas e a fitotoxicidade à cevada, já que são escassos os 
produtos existentes na atualidade para esse fim. Aliado às poucas opções disponíveis de herbicidas no mercado, soma-se ainda, na atualidade, a ocorrência de azevém resistente aos herbicidas inibidores de ALS, ACCase e EPSPs e nabo resistente a ALS, aumentando ainda mais a dificuldade da adoção do método químico de controle em cevada, já que esses mecanismos de ação são os mais utilizados para o manejo de plantas daninhas em cereais de inverno.

Desse modo objetivou-se com o trabalho, identificar possíveis alternativas para o manejo químico de azevém, bem como verificar a fitotoxicidade destes herbicidas sobre os componentes de rendimento de dois genótipos de cevada.

\section{Material e Métodos}

O experimento foi conduzido a campo, em área experimental do Colégio Agrícola Estadual Ângelo Emílio Grando, em Erechim/RS, durante a estação de crescimento 2012/13. O solo da área experimental é classificado como Latossolo Vermelho Aluminoférrico húmico, Unidade de mapeamento Erechim (Embrapa, 2013). A semeadura da cevada foi realizada em sistema de plantio direto, sendo que 15 dias antes dessa operação efetuou-se a dessecação da vegetação com o herbicida glyphosate na dose de $1.080 \mathrm{~g}$ $\mathrm{ha}^{-1}$ de equivalente ácido. A correção do $\mathrm{pH}$ e a adubação do solo foram realizadas de acordo com a análise físico-química, seguindo-se as recomendações técnicas de adubação para a cultura (ROLAS, 2004). A adubação química, na base, foi de $230 \mathrm{~kg} \mathrm{ha}^{-1}$ da fórmula 05-30-15 de N-P-K. Em cobertura aplicou-se adubação nitrogenada em duas épocas, sendo a primeira no estádio de perfilhamento e a segunda no elongamento, nas doses de $112,5 \mathrm{~kg} \mathrm{ha}^{-1} \mathrm{de}$ ureia, em cada estádio.

Cada unidade experimental foi composta por uma área de $11,05 \mathrm{~m}^{2}(5 \times 2,21 \mathrm{~m})$, onde foram semeados individualmente os genótipos de cevada MN610 e Crioula, com espaçamento entrelinhas de $0,17 \mathrm{~m}$ e densidade final de 300 plantas $\mathrm{m}^{-2}$.

$\mathrm{O}$ experimento foi instalado em delineamento de bloco casualizados, arranjados, em esquema fatorial 2 x 12 (genótipo $\mathrm{x}$ herbicidas), com quatro repetições. No fator A foram alocados os genótipos de cevada (MN610 e Crioula) e no B os herbicidas (iodosulfuron $0,100 \mathrm{~kg} \mathrm{ha}^{-1}$; imazethapyr + imazapic $-1,250 \mathrm{~L}$ $\mathrm{ha}^{-1}$; clomazone - $0,800 \mathrm{~L} \mathrm{ha}^{-1}$; propanil 6,000 $\mathrm{kg} \mathrm{ha}^{-1}$; oxyfluorfen - 1,000 $\mathrm{L} \mathrm{ha}^{-1}$; metsulfuronmethyl - 0,0033 kg ha-1; 2,4-D - 1,250 $\mathrm{L} \mathrm{ha}^{-1}$; cyhalofop-p-buthyl - 1,380 $\mathrm{L} \mathrm{ha}^{-1}$; penoxsulam - 0,175 $\mathrm{L} \mathrm{ha}^{-1}$; pyroxsulam - 0,400 L ha-1, mais duas testemunhas uma capinada e outra infestada.

Cada herbicida recebeu o adjuvante recomendado pelo respectivo fabricante. Os herbicidas clomazone e oxyfluorfen foram aplicados em pré-emergência e, todos os demais, em pós-emergência da cultura, quando essa estava com quatro folhas a dois perfilhos e do azevém no estádio de duas a quatro folhas. A densidade de azevém na área experimental era de 50 plantas $\mathrm{m}^{-2}$. A aplicação dos herbicidas foi efetuada com pulverizador costal de precisão, pressurizado a $\mathrm{CO}_{2}$, equipado com quatro pontas de pulverização tipo leque DG 110.02, sob pressão constante de $2,0 \mathrm{kgf} \mathrm{\textrm {cm } ^ { - 2 }}$ e velocidade de deslocamento de $3,6 \mathrm{~km} \mathrm{~h}^{-1}$, o que proporcionou a vazão de $150 \mathrm{~L} \mathrm{ha}^{-1}$ de calda de herbicida. As condições ambientais no momento da aplicação dos herbicidas em pré e pós-emergência estão descritas na Tabela 1.

As avaliações de controle foram realizadas visualmente, atribuindo-se notas percentuais de 0 a $100 \%$, aos $7,14,21,28,35$ e 42 dias após a aplicação dos tratamentos (DAT), no florescimento e na pré-colheita da cultura, e as de fitotoxicidade aos 7, 14, 21, 28, 35 e 42 DAT. Para avaliar o controle e a fitotoxicidade dos herbicidas foram atribuídas notas zero $(0 \%)$ aos tratamentos com ausência de controle do azevém ou de fitotoxicidade à cultura e a nota cem $(100 \%)$ para controle total da planta daninha ou morte completa das plantas de 
cevada, de acordo a metodologia proposta pela SBCPD (1995).

Tabela 1. Condições ambientais no momento da aplicação dos tratamentos em pré e pósemergência. UFFS, Erechim, 2012/13.

\begin{tabular}{cccccc}
\hline $\begin{array}{c}\text { Modalidade } \\
\text { de aplicação }\end{array}$ & $\begin{array}{c}\text { Lum. } \\
\%\end{array}$ & $\begin{array}{c}\text { Temp. } \\
{ }^{\circ} \mathrm{C}\end{array}$ & $\begin{array}{c}\text { U.R. } \\
(\%)\end{array}$ & Solo & $\begin{array}{c}\text { V.V. } \\
\mathrm{km} \mathrm{h}^{-1}\end{array}$ \\
\hline PRÉ & 100 & 17 & 79 & Úmido & 04 \\
PÓS & 100 & 19 & 78 & Úmido & 03 \\
\hline Lum = Luminosidade; Temp = Temperatura; U.R. = Umidade relativa \\
do ar; Solo = Condições de umidade; V.V. = Velocidade do vento.
\end{tabular}

$\mathrm{Na}$ pré-colheita da cevada foi determinado o número de espigas em área de $0,25 \mathrm{~m}^{2}$ no centro de cada unidade experimental. Para determinar o comprimento de espigas $(\mathrm{cm})$, o número de grãos cheios, o número de grãos estéreis e o total de grãos por espiga foram colhidos aleatoriamente, em cada unidade experimental, 10 espigas dos genótipos de cevada, as quais foram acondicionadas em saco de papel e encaminhadas para o Laboratório de Sementes da Universidade Federal da Fronteira Sul (UFFS), Câmpus Erechim/RS, onde foram realizadas as avaliações.

A colheita dos genótipos de cevada foi realizada quando os grãos atingiram $15 \%$ de umidade, em área útil de $4,5 \mathrm{~m}^{2}$ por unidade experimental, efetuando-se posteriormente a trilha. Por fim determinou-se ainda a massa de mil grãos (g), contando-se 8 amostras de 100 grãos cada e pesando-se as mesmas em balança analítica. Para determinar a produtividade, a umidade dos grãos foi ajustada para $13 \%$ e os dados de produção extrapolados para $\mathrm{kg} \mathrm{ha}^{-1}$.

Os dados foram submetidos à análise de variância pelo teste $\mathrm{F}$ e, quando se observou efeito significativo, os mesmos foram submetidos ao teste de Tukey $(\mathrm{p} \leq 0,05)$ para comparação das médias dos tratamentos.

\section{Resultados e Discussão}

Para todas as variáveis respostas estudadas, verificou-se interação entre os fatores herbicidas versus genótipos de cevada.

Observou-se para o controle de azevém avaliado aos 07 DAT, para os dois genótipos de cevada (MN610 e Crioula), que o herbicida clomazone apresentou os melhores resultados, equivalendo-se a testemunha capinada (Tabela 2). Nessa primeira avaliação todos os demais herbicidas apresentaram baixos percentuais de controle. O clomazone, mesmo que tenha diminuído os índices de controle do azevém após os 07 DAT, ainda apresentou controle superior a $85 \%$ até os 21 DAT. Ressalta-se que $80 \%$ é o controle mínimo que determinado herbicida deve apresentar para ser recomendado para o controle de plantas daninhas em culturas (Oliveira et al., 2009), desse modo o clomazone poderia ser indicado para o controle do azevém infestante da cevada, principalmente nos casos onde essa planta daninha é resistente aos herbicidas inibidores de ALS, ACCase e EPSPs. Convém destacar que a brusca queda de controle do azevém pelo clomazone, saindo dos $91 \%$ aos 21 DAT para $0 \%$ aos 28 DAT deve-se principalmente ao novo fluxo de emergência da planta daninha em meio a cultura da cevada, já que essa planta daninha apresenta fluxos de emergência escalonado.

Os resultados demonstram que os herbicidas iodosulfuron, imazethapyr + imazapic e pyroxsulam apresentaram os melhores controles do azevém, equivalendo-se a testemunha capinada, aos 14, 21, 28, 35 e 42 DAT, bem como na avaliação de controle efetuada no estádio de florescimento dessa planta daninha e na pré-colheita da cevada (Tabelas 2 e 3). O bom desempenho desses produtos aplicados para o controle do azevém, do início do ciclo até a colheita da cevada, mostra que estes podem ser indicados para o controle dessa planta daninha.

Nas avaliações efetuadas observou-se que o controle de azevém, com o uso de cyhalofop-p-butyl apresentou eficiência, de modo geral, inferior ao constatado para iodosulfuron, imazethapyr + imazapic e pyroxsulam, porém ainda a níveis aceitáveis dos 14 até os 35 DAT e no florescimento da planta daninha (Tabelas 2 e 3). Cabe destacar que mesmo que esse produto tenha controlado $80 \%$ 
da população do azevém que infestou a cevada a recomendação do mesmo para o manejo químico dessa planta daninha torna-se preocupante, já que essa espécie é muito competitiva com a cultura e ainda é resiste aos mecanismos de ação inibidores da EPSPs, ALS e ACCase (Roman et al., 2004; Mariani et al., 2012; Vargas et al., 2013).

Tabela 2. Controle (\%) de azevém infestante de genótipos de cevada (MN610 e Criola) em função da aplicação de herbicidas. UFFS/Erechim/RS, 2012/13.

\begin{tabular}{|c|c|c|c|c|c|c|c|c|c|c|c|c|}
\hline \multirow{3}{*}{ Tratamentos } & \multicolumn{12}{|c|}{ Controle de azevém (\%) } \\
\hline & \multicolumn{2}{|c|}{7 DAT } & \multicolumn{2}{|c|}{14 DAT } & \multicolumn{2}{|c|}{$21 \mathrm{DAT}$} & \multicolumn{2}{|c|}{28 DAT } & \multicolumn{2}{|c|}{35 DAT } & \multicolumn{2}{|c|}{42 DAT } \\
\hline & MN610 & Criola & MN610 & Criola & MN610 & Criola & MN610 & Criola & MN610 & Criola & MN610 & Criola \\
\hline IOD & ${ }^{n s} 62 c^{1}$ & $55 \mathrm{~b}$ & ${ }^{\mathrm{ns}} 90 \mathrm{~b}$ & $92 a b c$ & ${ }^{\mathrm{ns}} 93 \mathrm{bc}$ & $97 \mathrm{ab}$ & ${ }^{\mathrm{n}} 100 \mathrm{a}$ & $99 \mathrm{a}$ & $* 97$ a & $91 \mathrm{bc}$ & ${ }^{\mathrm{n} n} 100 \mathrm{a}$ & $100 \mathrm{a}$ \\
\hline $\mathrm{IMA}+\mathrm{IMP}$ & $* 63 \mathrm{c}$ & $52 \mathrm{~b}$ & ${ }^{\text {ns9 } 93 a b}$ & $96 \mathrm{ab}$ & ${ }^{\text {ns} 99} \mathrm{ab}$ & $96 \mathrm{ab}$ & ${ }^{\mathrm{ns}} 92 \mathrm{~b}$ & $95 \mathrm{a}$ & ${ }^{\text {ns93 a }}$ & $93 a b c$ & ${ }^{n s} 87 \mathrm{~b}$ & $91 \mathrm{a}$ \\
\hline CLO & ${ }^{\text {ns }} 91 \mathrm{ab}$ & $89 a$ & ${ }^{\mathrm{ns}} 92 \mathrm{~b}$ & $86 \mathrm{c}$ & ${ }^{\text {ns } 90 ~ c ~}$ & $91 \mathrm{~b}$ & ${ }^{\mathrm{ns}} 0 \mathrm{c}$ & $0 \mathrm{c}$ & ${ }^{\mathrm{n}} 0 \mathrm{c}$ & $d$ & ${ }^{n s} 0 \mathrm{~d}$ & $0 \mathrm{c}$ \\
\hline PRO & ${ }^{n s} 9 \mathrm{e}$ & $10 \mathrm{c}$ & ${ }^{\mathrm{ns}} 7 \mathrm{~d}$ & $5 \mathrm{f}$ & ns $5 \mathrm{f}$ & $3 \mathrm{e}$ & ${ }^{\mathrm{n} s} 0 \mathrm{c}$ & $0 \mathrm{c}$ & ${ }^{\mathrm{ns}} 0 \mathrm{c}$ & $0 \mathrm{~cd}$ & ${ }^{\mathrm{ns}} 0 \mathrm{~d}$ & $0 \mathrm{c}$ \\
\hline OXY & $* 79 \mathrm{~b}$ & $47 \mathrm{~b}$ & $* 77 \mathrm{c}$ & $54 \mathrm{e}$ & ${ }^{\mathrm{n}} 63 \mathrm{e}$ & $57 \mathrm{~d}$ & ${ }^{\mathrm{ns}} 0 \mathrm{c}$ & $0 \mathrm{c}$ & ${ }^{\mathrm{ns}} 0 \mathrm{c}$ & $0 \mathrm{~cd}$ & ${ }^{\mathrm{ns}} 0 \mathrm{~d}$ & $0 \mathrm{c}$ \\
\hline MET & ${ }^{n s} 0 \mathrm{e}$ & $0 \mathrm{e}$ & ${ }^{\mathrm{ns}} 0 \mathrm{~d}$ & $0 \mathrm{f}$ & ${ }^{n s} 0 \mathrm{f}$ & $0 \mathrm{e}$ & ${ }^{\mathrm{n}} 0 \mathrm{c}$ & $0 \mathrm{c}$ & ${ }^{\mathrm{n}} 0 \mathrm{c}$ & $0 \mathrm{~cd}$ & ${ }^{\mathrm{ns}} 0 \mathrm{~d}$ & $0 \mathrm{c}$ \\
\hline 2,4-D & ${ }^{n n} 0 \mathrm{e}$ & $0 \mathrm{e}$ & ${ }^{\mathrm{ns}} 0 \mathrm{~d}$ & $0 \mathrm{f}$ & ${ }^{n s} 0 \mathrm{f}$ & $0 \mathrm{e}$ & ${ }^{\mathrm{n} s} 0 \mathrm{c}$ & $0 \mathrm{c}$ & ${ }^{\mathrm{ns}} 0 \mathrm{c}$ & $0 \mathrm{~cd}$ & ${ }^{n s} 0 \mathrm{~d}$ & $0 \mathrm{c}$ \\
\hline CYH & ${ }^{n s} 48 \mathrm{~d}$ & $47 \mathrm{~b}$ & ${ }^{\mathrm{ns}} 88 \mathrm{~b}$ & $91 \mathrm{bc}$ & ns $96 a b c$ & $95 \mathrm{ab}$ & ${ }^{\mathrm{ns}} 92 \mathrm{~b}$ & $86 \mathrm{~b}$ & *93 a & $87 \mathrm{c}$ & $* 67 \mathrm{c}$ & $53 \mathrm{~b}$ \\
\hline PEN & ${ }^{\mathrm{n}} 55 \mathrm{~cd}$ & $47 \mathrm{~b}$ & ${ }^{\text {ns }} 77 \mathrm{c}$ & $77 \mathrm{~d}$ & $* 79 \mathrm{~d}$ & $74 \mathrm{c}$ & ${ }^{\mathrm{ns}} 0 \mathrm{c}$ & $0 \mathrm{c}$ & ${ }^{\mathrm{n}} 0 \mathrm{c}$ & $0 \mathrm{~d}$ & ${ }^{n s} 0 \mathrm{~d}$ & $0 \mathrm{c}$ \\
\hline PYR & ${ }^{\mathrm{n}} 62 \mathrm{c}$ & $56 \mathrm{~b}$ & ns93 ab & $94 a b c$ & ns99 ab & $98 \mathrm{ab}$ & ${ }^{\mathrm{ns}} 100 \mathrm{a}$ & $98 \mathrm{a}$ & ${ }^{\mathrm{ns}} 100 \mathrm{a}$ & $96 a b$ & ${ }^{\mathrm{ns}} 100 \mathrm{a}$ & $99 \mathrm{a}$ \\
\hline Test & ${ }^{\mathrm{n} s} 100 \mathrm{a}$ & $100 \mathrm{a}$ & ${ }^{\mathrm{n} s} 100 \mathrm{e}$ & $100 \mathrm{a}$ & ${ }^{\mathrm{n} s} 100 \mathrm{a}$ & $100 \mathrm{a}$ & ${ }^{\mathrm{ns}} 100 \mathrm{a}$ & $100 \mathrm{a}$ & ${ }^{\mathrm{n} s} 100 \mathrm{a}$ & $100 \mathrm{a}$ & ${ }^{\mathrm{n} s} 100 \mathrm{a}$ & $100 \mathrm{a}$ \\
\hline Test. i & ${ }^{\mathrm{ns}} 0 \mathrm{e}$ & $0 \mathrm{e}$ & ${ }^{\mathrm{ns}} 0 \mathrm{~d}$ & $0 \mathrm{Af}$ & ${ }^{\mathrm{ns}} 0 \mathrm{f}$ & $0 \mathrm{e}$ & $0 \mathrm{c}$ & $0 \mathrm{c}$ & ${ }^{\mathrm{ns}} 0 \mathrm{c}$ & $0 \mathrm{~d}$ & ${ }^{\mathrm{ns}} 0 \mathrm{~d}$ & $0 \mathrm{c}$ \\
\hline CV (\%) & 6,25 & 6,25 & 4,25 & 4,25 & 3,93 & 3,93 & 4,22 & 4,22 & 4,48 & 4,48 & 3,91 & 3,91 \\
\hline
\end{tabular}

IOD = iodosulfuron; IMA + IMP = imazethapyr + imazapic; CLO = clomazone; PRO = propanil; OXY = oxyfluorfen; MET = metsulfuron-methyl; $\mathrm{CYH}=$ cyhalofop-p-buthyl; $\mathrm{PEN}=$ penoxsulam; $\mathrm{PYR}=$ pyroxsulam. ${ }^{1}$ Médias seguidas de letras minúsculas distintas, na coluna, diferem entre $\mathrm{si}$ pelo teste de Tukey $(\mathrm{p} \leq 0,05)$ e por *, na linha, entre os genótipos de cevada para cada variável resposta, diferem entre si pelo teste $\mathrm{t}(\mathrm{p} \leq 0,05)$.

Destaca-se que a campo, pode-se usar cultivares que apresentem características morfológicas que demonstrem, por exemplo, maior índice de área foliar, estatura de plantas, dentre outras, que irão sombrear o solo e desse modo auxiliar os herbicidas no controle de plantas daninhas. Nesse estudo observou-se diferenciação dos genótipos avaliados, pois aos 07 DAT houve menor controle do azevém ao se aplicar o oxyfluorfen e o imazethapyr + imazapic sobre o genótipo Crioula (Tabela 2). Já aos 14 e 21 DAT o uso de oxyfluorfen, nesse mesmo genótipo de cevada, novamente apresentou os piores resultados para o controle da planta daninha. $\mathrm{O}$ penoxsulam aplicado para o controle de azevém aos 21 DAT demonstrou inferioridade quando aplicado sobre o genótipo Crioula, sendo o mesmo fato constatado para o cyhalofop-p-butyl aos 35 e 42 DAT. Isto significa que o controle do azevém com estes herbicidas ocorre com maior intensidade quando se utiliza o genótipo MN610. O maior controle observado para o azevém ao infestar o genótipo MN610 deve-se ao fato de que esse apresentou maior estatura de plantas, perfilhamento e índice área foliar se comparado à Crioula (dados não apresentados), sombreando a planta daninha presente na lavoura e desse modo ocasionando melhor controle. De acordo com Balbinot Jr. et al., (2003) esse fato decorre em função das características morfofisiológicas de cada genótipo, que define a capacidade em competir com as plantas daninhas pelos recursos disponíveis no ambiente, em especial a luz.

Com relação à fitotoxicidade dos herbicidas ocasionada sobre a cultura observouse que a mistura comercial de imazethapyr + imazapic e o cyhalofop-p-butyl foram os produtos que mais danos ocasionaram aos dois genótipos de cevada em todas as épocas avaliadas (Tabela 4). A fitotoxicidade avaliada aos $07,14,21$ e 28 DAT demonstrou que os herbicidas imazethapyr + imazapic, clomazone, cyhalofop-p-buthyl e pyroxsulam apresentaram os maiores sintomas de injúrias sobre as plantas dos genótipos MN610 e Crioula. Os demais herbicidas apresentaram sintomas de fitotoxicidade muito semelhantes ou mesmo em muitas situações se igualaram a testemunha 
capinada, ou até menores que as injúrias de imazethapyr + imazapic, clomazone, cyhalofopp-buthyl e pyroxsulam. Cabe destacar que após os 28 DAT as plantas dos genótipos de cevada que apresentaram baixos sintomas de fitotoxicidade recuperaram-se dos danos ocasionados pelos herbicidas, porém após essa avaliação, aos 35 e 42 DAT, o imazethapyr + imazapic e o clomazone continuaram ocasionando as maiores injúrias à cultura. Os sintomas de fitotoxicidade persistiram por um tempo relativamente longo, até os 28 DAT, especula-se que isso seja em função de que o metabolismo das culturas com temperaturas mais baixas, no inverno, demora mais tempo para eliminar ou metabolizar as injúrias ocasionadas pelos herbicidas. Vargas \& Roman (2005) também constaram efeitos similares, aos observados no presente trabalho, ao avaliarem a seletividade de diferentes herbicidas aplicados sobre cereais de inverno.

Tabela 3. Controle (\%) de azevém infestante de genótipos de cevada (MN610 e Crioula) com uso de herbicidas no florescimento da cultura e da planta daninha, e na pré-colheita da cultura. UFFS/Erechim/RS, 2012/13.

\begin{tabular}{|c|c|c|c|c|}
\hline \multirow{2}{*}{ Tratamentos } & \multicolumn{2}{|c|}{ Florescimento } & \multicolumn{2}{|c|}{ Pré-colheita } \\
\hline & MN610 & Crioula & MN610 & Crioula \\
\hline Iodosulfuron & ${ }^{\mathrm{ns}} 100,0 \mathrm{a}^{1}$ & $99,0 \mathrm{ab}$ & ${ }^{\mathrm{n}} 98,5 \mathrm{a}$ & $99,3 \mathrm{a}$ \\
\hline Imazethapyr + imazapic & ${ }^{\mathrm{ns}} 94,8 \mathrm{~b}$ & $94,7 \mathrm{~b}$ & $* 76,3 \mathrm{c}$ & $84,3 \mathrm{~b}$ \\
\hline Clomazone & ${ }^{\mathrm{ns}} 0,0 \mathrm{c}$ & $0,0 \mathrm{e}$ & $* 33,8 \mathrm{e}$ & $23,3 \mathrm{~d}$ \\
\hline Propanil & ${ }^{\mathrm{ns}} 0,0 \mathrm{c}$ & $0,0 \mathrm{e}$ & ${ }^{\mathrm{ns}} 0,0 \mathrm{f}$ & $0,0 \mathrm{e}$ \\
\hline Oxyfluorfen & ${ }^{\mathrm{ns}} 0,0 \mathrm{c}$ & $0,0 \mathrm{e}$ & $* 0,0 \mathrm{f}$ & $55,0 \mathrm{c}$ \\
\hline Metsulfuron-methyl & ${ }^{\mathrm{ns}} 0,0 \mathrm{c}$ & $0,0 \mathrm{e}$ & ${ }^{\mathrm{ns}} 0,0 \mathrm{Af}$ & 0,0 \\
\hline $2,4-\mathrm{D}$ & ${ }^{\mathrm{ns}} 0,0 \mathrm{c}$ & $0,0 \mathrm{e}$ & ${ }^{\mathrm{ns}}, 0 \mathrm{f}$ & $0,0 \mathrm{e}$ \\
\hline Cyhalofop-p-buthyl & $* 91,7 \mathrm{~b}$ & $85,0 \mathrm{c}$ & $* 61,2 \mathrm{~d}$ & $20,0 \mathrm{~d}$ \\
\hline Penoxsulam & $* 0,0 \mathrm{Bc}$ & $79,0 \mathrm{~d}$ & $* 86,0 \mathrm{~b}$ & $57,3 \mathrm{c}$ \\
\hline Pyroxsulam & ns $100,0 \mathrm{a}$ & $97,8 \mathrm{ab}$ & ${ }^{\mathrm{ns}} 98,5 \mathrm{a}$ & $98,5 \mathrm{a}$ \\
\hline Testemunha capinada & ns $100,0 \mathrm{a}$ & $100,0 \mathrm{a}$ & ${ }^{\mathrm{ns}} 100,0 \mathrm{Aa}$ & $100,0 \mathrm{a}$ \\
\hline Testemunha infestada & ${ }^{\mathrm{ns}} 0,0 \mathrm{c}$ & $0,0 \mathrm{e}$ & ${ }^{\mathrm{ns}} 0,0 \mathrm{Af}$ & $0,0 \mathrm{e}$ \\
\hline $\mathrm{CV}$ & 4,32 & 4,32 & 5,57 & 5,57 \\
\hline
\end{tabular}

Ao se comparar os genótipos de cevada entre si com relação a fitotoxicidade, observouse que o Crioula foi menos suscetível aos herbicidas iodosulfuron e imazethapyr + imazapic, aos 14, 21, 28 e 42 DAT (Tabela 4). O genótipo MN610 apresentou menor fitotoxicidade que o Crioula para o cyhalofopp-buthyl nas avaliações efetuadas aos 07, 14 e 21 DAT. De modo geral, o genótipo MN610 apresentou-se mais sensível aos herbicidas aplicados, se comparado a Crioula em todas as épocas avaliadas.

A cultura da cevada é considerada extremamente sensível a aplicação de herbicidas utilizados para o controle de plantas daninhas. Nunes et al., (2007) constataram que o metribuzin promoveu cerca de $90 \%$ de fitotoxicidade e que atrazine e cloransulfuron não causaram injúrias a cevada nas avaliações efetuadas aos 14 e 21 DAT. Os mesmos autores concluíram que a cevada apresentou tolerância aos herbicidas atrazine e cloransulfuron se comparados a outros tratamentos.

Os resultados demonstram maior comprimento de espigas para ambos os genótipos de cevadas ao se aplicar o cyhalofopp-buthyl, porém não diferindo do iodosulfuron, imazethapyr + imazapic, clomazone, metsulfuron-methyl, 2,4-D, penoxsulam e da testemunha capinada para o genótipo MN610, e do imazapic + imazethapyr para o Crioula (Tabela 5). Ao se comparar os genótipos de cevada dentro de cada herbicida aplicado observou-se que iodosulfuron, imazethapyr + imazapic, propanil, oxyfluorfen, cyhalofop-pbuthyl e pyroxsulam apresentaram maior comprimento de espigas para o Crioula. O genótipo MN610 foi estatisticamente superior 
ao Crioula somente para o 2,4-D. Os demais tratamentos avaliados apresentaram comprimento de espigas que não diferiram entre si. Tottman (1977) ao avaliar o efeito dos herbicidas 2,4-D e MCPA, em aplicações precoces, observou influência negativa sobre a formação de espigas, por interferência nos primórdios de espiguetas no ápice de crescimento em plantas de trigo. Esse resultado assemelha-se ao constatado no presente trabalho ao se aplicar diferentes herbicidas para o controle de plantas daninhas infestantes dos dois genótipos de cevada.

Tabela 4. Fitotoxicidade (\%) à genótipos de cevada em função da aplicação de herbicidas. UFFS/Erechim/RS, 2012/13.

\begin{tabular}{|c|c|c|c|c|c|c|c|c|c|c|c|c|}
\hline \multirow{3}{*}{ Tratamentos } & \multicolumn{12}{|c|}{ Fitotoxicidade a genótipos de cevada (\%) } \\
\hline & \multicolumn{2}{|c|}{$7 \mathrm{DAT}$} & \multicolumn{2}{|c|}{14 DAT } & \multicolumn{2}{|c|}{$21 \mathrm{DAT}$} & \multicolumn{2}{|c|}{28 DAT } & \multicolumn{2}{|c|}{$35 \mathrm{DAT}$} & \multicolumn{2}{|c|}{$42 \mathrm{DAT}$} \\
\hline & MN610 & Criola & MN610 & Criola & MN610 & Criola & MN610 & Criola & MN610 & Criola & MN610 & Criola \\
\hline IOD & ${ }^{\mathrm{ns}} 8,0 \mathrm{~cd}^{1}$ & $9,0 \mathrm{c}$ & $* 8,8 \mathrm{~d}$ & $5,5 \mathrm{e}$ & $* 13,0 \mathrm{e}$ & $9,8 \mathrm{~d}$ & $* 33,0 \mathrm{bc}$ & $15,0 \mathrm{de}$ & ${ }^{\mathrm{ns}} 6,0 \mathrm{~cd}$ & $4,0 \mathrm{~b}$ & $* 13,0 \mathrm{c}$ & $1,0 \mathrm{~b}$ \\
\hline IMA + IMP & ns $27,0 \mathrm{~b}$ & $26,0 \mathrm{~b}$ & $* 65,0$ a & $61,3 \mathrm{~b}$ & $* 91,5 \mathrm{a}$ & 86,3 a & $* 98,0$ a & $85,0 \mathrm{a}$ & $* 89,0 \mathrm{a}$ & 80,0 a & $* 80,0 \mathrm{a}$ & $65,0 \mathrm{a}$ \\
\hline CLO & $* 63,0 \mathrm{a}$ & $52,0 \mathrm{a}$ & $* 55,5 \mathrm{~b}$ & $55,0 \mathrm{c}$ & ${ }^{\mathrm{n}} 53,3 \mathrm{c}$ & $56,3 \mathrm{~b}$ & ${ }^{\mathrm{ns}} 43,0 \mathrm{~b}$ & $52,0 \mathrm{~b}$ & ${ }^{\mathrm{ns}} 8,0 \mathrm{~cd}$ & $11,0 \mathrm{~b}$ & $* 16,0 \mathrm{c}$ & $1,0 \mathrm{~b}$ \\
\hline PRO & ${ }^{\mathrm{n}} 3,0 \mathrm{~cd}$ & $5,0 \mathrm{~cd}$ & ${ }^{\mathrm{ns}} 2,5 \mathrm{e}$ & 2,5 ef & ${ }^{\mathrm{ns}} 2,8 \mathrm{f}$ & $2,8 \mathrm{e}$ & $* 31,0 \mathrm{bcd}$ & $17,0 \mathrm{~d}$ & ${ }^{\mathrm{ns}} 10,0 \mathrm{~cd}$ & $5,0 \mathrm{~b}$ & ${ }^{\mathrm{ns}} 0,0 \mathrm{~d}$ & $1,0 \mathrm{~b}$ \\
\hline OXY & ${ }^{\mathrm{ns}} 3,0 \mathrm{~cd}$ & $4,0 \mathrm{~cd}$ & ${ }^{\mathrm{ns}} 2,5 \mathrm{e}$ & 2,5 ef & ${ }^{\mathrm{ns}} 2,0 \mathrm{f}$ & $3,0 \mathrm{e}$ & ${ }^{\mathrm{ns}} 13,0$ ef & $13,0 \mathrm{de}$ & ${ }^{\mathrm{ns}} 3,0 \mathrm{~cd}$ & $4,0 \mathrm{~b}$ & ${ }^{\mathrm{ns}} 0,0 \mathrm{~d}$ & $1,0 \mathrm{~b}$ \\
\hline MET & $* 4,0 \mathrm{~cd}$ & $9,0 \mathrm{c}$ & ${ }^{\mathrm{ns}} 2,3 \mathrm{e}$ & 4,5 ef & ${ }^{\mathrm{ns}} 2,8 \mathrm{f}$ & $3,8 \mathrm{e}$ & ${ }^{\mathrm{ns}} 17,0$ cde & $15,0 \mathrm{de}$ & ${ }^{\mathrm{ns}} 9,0 \mathrm{~cd}$ & $8,0 \mathrm{~b}$ & ${ }^{\mathrm{ns}} 0,0 \mathrm{~d}$ & $2,0 \mathrm{~b}$ \\
\hline 2,4-D & ${ }^{\mathrm{ns}} 6,0 \mathrm{~cd}$ & $8,0 \mathrm{~cd}$ & ${ }^{\mathrm{ns}} 2,8 \mathrm{e}$ & 2,8 ef & $* 0,3 \mathrm{f}$ & $3,5 \mathrm{e}$ & $* 3,0$ ef & $15,0 \mathrm{de}$ & ${ }^{\mathrm{ns}} 3,0 \mathrm{~cd}$ & $5,0 \mathrm{~b}$ & ${ }^{\mathrm{ns}} 0,0 \mathrm{~d}$ & $0,0 \mathrm{~b}$ \\
\hline $\mathrm{CYH}$ & $* 11,0 \mathrm{c}$ & $27,0 \mathrm{~b}$ & $* 60,0 \mathrm{~b}$ & $66,3 \mathrm{a}$ & $* 79,3 \mathrm{~b}$ & $88,8 \mathrm{a}$ & ${ }^{\mathrm{ns}} 87,0 \mathrm{a}$ & 86,0 a & ns $75,0 \mathrm{~b}$ & $81,0 \mathrm{a}$ & $* 48,0 \mathrm{~b}$ & $64,0 \mathrm{a}$ \\
\hline PEN & ${ }^{\mathrm{ns}} 6,0 \mathrm{~cd}$ & $10,0 \mathrm{c}$ & ${ }^{\mathrm{ns}} 2,8 \mathrm{e}$ & 3,3 ef & ns $3,8 \mathrm{f}$ & 5,3 de & $* 15,0$ def & $29,0 \mathrm{~cd}$ & $* 3,0 \mathrm{~cd}$ & $11,0 \mathrm{~b}$ & ${ }^{\mathrm{ns}} 0,0 \mathrm{~d}$ & $2,0 \mathrm{~b}$ \\
\hline PYR & ${ }^{\mathrm{ns}} 11,0 \mathrm{c}$ & $11,0 \mathrm{c}$ & $* 38,3 \mathrm{c}$ & $43,5 \mathrm{~d}$ & ${ }^{\mathrm{ns}} 40,8 \mathrm{~d}$ & $42,5 \mathrm{c}$ & $37,0 \mathrm{~b}$ & $42,0 \mathrm{bc}$ & ${ }^{\mathrm{ns}} 15,0 \mathrm{c}$ & $9,0 \mathrm{~b}$ & ${ }^{\mathrm{ns}} 10,0 \mathrm{~cd}$ & $3,0 \mathrm{~b}$ \\
\hline Test. c & ${ }^{\mathrm{ns}} 0,0 \mathrm{~d}$ & $0,0 \mathrm{~d}$ & ${ }^{\mathrm{ns}} 0,0 \mathrm{e}$ & $0,0 \mathrm{f}$ & ${ }^{\mathrm{ns}} 0,0 \mathrm{f}$ & $0,0 \mathrm{e}$ & ${ }^{\mathrm{ns}} 0,0 \mathrm{f}$ & $0,0 \mathrm{e}$ & ${ }^{\mathrm{ns}} 0,0 \mathrm{~d}$ & $0,0 \mathrm{~b}$ & ${ }^{\mathrm{ns}} 0,0 \mathrm{~d}$ & $0,0 \mathrm{~b}$ \\
\hline Test. infestada & ${ }^{\mathrm{ns}} 0,0 \mathrm{~d}$ & $0,0 \mathrm{~d}$ & ${ }^{\mathrm{ns}} 0,0 \mathrm{e}$ & $0,0 \mathrm{f}$ & ${ }^{\mathrm{ns}} 0,0 \mathrm{f}$ & $0,0 \mathrm{e}$ & ${ }^{\mathrm{ns}} 0,0 \mathrm{f}$ & $0,0 \mathrm{e}$ & ${ }^{\mathrm{ns}} 0,0 \mathrm{~d}$ & $0,0 \mathrm{~b}$ & ${ }^{\mathrm{ns}} 0,0 \mathrm{~d}$ & $0,0 \mathrm{~b}$ \\
\hline $\mathrm{CV}(\%)$ & 12,08 & 12,08 & 9,83 & 9,83 & 9,07 & 9,07 & 9,06 & 9,06 & 12,56 & 12,56 & 15,32 & 15,32 \\
\hline
\end{tabular}

IOD = iodosulfuron; IMA + IMP = imazethapyr + imazapic; CLO = clomazone; PRO = propanil; OXY = oxyfluorfen; MET = metsulfuron-methyl; $\mathrm{CYH}=$ cyhalofop-p-buthyl; PEN = penoxsulam; $\mathrm{PYR}=$ pyroxsulam. ${ }^{1}$ Médias seguidas de letras minúsculas distintas, na coluna, diferem entre si pelo teste de Tukey $(\mathrm{p} \leq 0,05)$ e por *, na linha, entre os genótipos de cevada para cada variável resposta, diferem entre si pelo teste $t(p \leq 0,05)$.

Ao se avaliar o número de espigas constatou-se resposta distinta entre os genótipos de cevada, quando submetidos à aplicação dos herbicidas (Tabela 5). Observou-se para o MN610 que os herbicidas penoxsulam e propanil não diferiram estatisticamente da testemunha capinada apresentando o maior número de espigas. Já para o Crioula os resultados demonstram que a aplicação de iodosulfuron, propanil e pyroxsulam equivalem-se entre si, sendo os melhores tratamentos quando comparado aos demais. Em comparação entre os genótipos, a Crioula só diferiu estatisticamente da MN610, com maior número de espigas, quando se aplicou o cyhalofop-p-buthyl, nos demais tratamentos não houve diferença ou a MN610 sobressaiu-se em relação a Crioula. Esse resultado pode estar relacionado ao efeito fitotóxico do herbicida sobre a cultura que vem a comprometer os componentes de rendimento. De acordo com Dal Magro et al. (2006) ao aplicarem imazethapyr + imazapic em diferentes cultivares de arroz (IRGA 417, BR-IRGA 410, BRS Pelotas e Qualimax 1), observaram elevado efeito fitotóxico sobre a cultura afetando negativamente os componentes de rendimento do arroz e consequentemente a produtividade de grãos. Contrariamente ao observado na presente pesquisa, Rodrigues et al. (2006) ao estudarem o comportamento do herbicida dicamba aplicado em diferentes épocas e doses sobre as cultivares de trigo (BR 23 e Embrapa 16), constataram em dois anos agrícolas (1992 e 1993) que o número de espigas não foi afetado por nenhum tratamento.

O clomazone e o oxyfluorfen foram os herbicidas que apresentaram o maior número de grãos estéreis do genótipo MN610 em comparação aos demais tratamentos, sendo inferiores inclusive a testemunha infestada (Tabela 5). Ao se comparar os genótipos entre si observou-se que o clomazone ocasionou 55,7\% mais grãos estéreis no MN610 em relação ao Crioula. O genótipo Crioula apresentou os piores resultados ao se aplicar o iodosulfuron, 
imazethapyr + imazapic e cyhalofop-pbutyl. O uso de pyroxsulam demonstrou, de modo geral, o menor número de grãos estéreis para os dois genótipos de cevada avaliados.

Tabela 5. Componentes de rendimento de grãos de genótipos de cevada em função da aplicação de herbicidas. UFFS, Erechim/RS, 2012/13.

\begin{tabular}{|c|c|c|c|c|c|c|c|c|c|c|}
\hline \multirow[t]{2}{*}{ Tratamentos } & \multicolumn{2}{|c|}{$\begin{array}{l}\text { Comprimento } \\
\text { da espiga }(\mathrm{cm})\end{array}$} & \multicolumn{2}{|c|}{ N. espigas $\left(\mathrm{m}^{2}\right)$} & \multicolumn{2}{|c|}{$\begin{array}{c}\text { N. grãos } \\
\text { estéreis/espiga } \\
\end{array}$} & \multicolumn{2}{|c|}{$\begin{array}{c}\text { N. grãos } \\
\text { cheios/espiga } \\
\end{array}$} & \multicolumn{2}{|c|}{$\begin{array}{c}\text { N. grãos } \\
\text { totais/espiga }\end{array}$} \\
\hline & MN610 & Crioula & MN610 & Crioula & MN610 & Crioula & MN610 & Crioula & MN610 & Crioula \\
\hline IOD & $* 7,8 \mathrm{abc}^{1}$ & $8,4 \mathrm{bc}$ & ${ }^{\mathrm{ns}} 1096,0 \mathrm{~b}$ & $1126,3 \mathrm{a}$ & $* 4,1 \mathrm{fg}$ & $6,4 \mathrm{a}$ & $* 19,4 \mathrm{c}$ & $22,0 \mathrm{~b}$ & $* 23,5 \mathrm{de}$ & $28,4 \mathrm{a}$ \\
\hline IMA + IMP & $* 8,2 \mathrm{a}$ & $8,8 \mathrm{ab}$ & $* 494,0 \mathrm{~g}$ & $280,0 \mathrm{~g}$ & ${ }^{\mathrm{ns}} 4,6 \mathrm{f}$ & $5,0 \mathrm{~b}$ & $* 20,8 \mathrm{~b}$ & $19,1 \mathrm{~d}$ & $* 25,4 \mathrm{bc}$ & $24,1 \mathrm{e}$ \\
\hline CLO & ${ }^{\mathrm{ns}} 8,0 \mathrm{ab}$ & $8,0 \mathrm{~cd}$ & $* 986,0 \mathrm{de}$ & 749,7 ef & $* 11,1 \mathrm{a}$ & $4,9 \mathrm{bc}$ & $* 15,8$ ef & $21,4 \mathrm{bc}$ & $26,9 \mathrm{a}$ & $26,3 \mathrm{~b}$ \\
\hline PRO & $* 7,1 \mathrm{c}$ & $8,0 \mathrm{~cd}$ & $* 1131,5 \mathrm{ab}$ & $1088,0 \mathrm{a}$ & $* 4,6$ ef & $4,0 \mathrm{de}$ & $* 18,6 \mathrm{c}$ & $20,5 \mathrm{c}$ & $* 23,2 \mathrm{e}$ & $24,5 \mathrm{de}$ \\
\hline OXY & ns7,7 abc & $7,5 \mathrm{~d}$ & $* 975,0 \mathrm{de}$ & $824,0 \mathrm{~cd}$ & $* 6,6 \mathrm{~cd}$ & $4,9 \mathrm{bc}$ & $* 15,1 \mathrm{f}$ & $17,8 \mathrm{e}$ & $* 21,8 \mathrm{f}$ & $22,7 \mathrm{f}$ \\
\hline MET & $* 7,2 \mathrm{c}$ & $8,4 \mathrm{bc}$ & $* 1099,0 \mathrm{~b}$ & $725,1 \mathrm{f}$ & $* 8,2 \mathrm{~b}$ & $3,6 \mathrm{e}$ & $* 17,1 \mathrm{~d}$ & $21,9 \mathrm{~b}$ & ${ }^{\mathrm{ns}} 25,4 \mathrm{bc}$ & $25,5 \mathrm{bcd}$ \\
\hline 2,4-D & $* 7,8 \mathrm{abc}$ & $7,3 \mathrm{~d}$ & $* 954,7 \mathrm{e}$ & 810,3 cde & $* 6,4 \mathrm{~d}$ & $4,8 \mathrm{bcd}$ & $* 19,1 \mathrm{c}$ & $15,6 \mathrm{f}$ & $* 25,6 \mathrm{~b}$ & $20,3 \mathrm{~g}$ \\
\hline CYH & $* 8,3 \mathrm{a}$ & $9,1 \mathrm{a}$ & $* 566,7 \mathrm{f}$ & $852,0 \mathrm{bcd}$ & $* 3,7 \mathrm{~g}$ & $5,3 \mathrm{~b}$ & $* 22,6 \mathrm{a}$ & $23,8 \mathrm{a}$ & $* 26,3 \mathrm{ab}$ & $29,0 \mathrm{a}$ \\
\hline PEN & ns7,7 abc & $7,5 \mathrm{~d}$ & $* 1133,3 \mathrm{ab}$ & $903,0 \mathrm{~b}$ & $* 7,2 \mathrm{~cd}$ & 4,2 cde & $* 16,8$ de & $17,5 \mathrm{e}$ & $* 24,0 \mathrm{de}$ & $21,7 \mathrm{f}$ \\
\hline PYR & $* 7,2 \mathrm{c}$ & $8,4 \mathrm{bc}$ & ${ }^{\mathrm{ns}} 1091,8 \mathrm{bc}$ & $1127,5 \mathrm{a}$ & ${ }^{\mathrm{ns}} 3,7 \mathrm{~g}$ & $3,8 \mathrm{e}$ & $* 20,7 \mathrm{~b}$ & $21,3 \mathrm{bc}$ & $* 24,3 \mathrm{~cd}$ & 25,1 cde \\
\hline Test. cap. & ${ }^{\mathrm{ns}} 8,1 \mathrm{ab}$ & $8,0 \mathrm{~cd}$ & $* 1177,5$ a & $875,0 \mathrm{bc}$ & $* 5,4 \mathrm{e}$ & 4,1 cde & ${ }^{\mathrm{ns}} 21,6 \mathrm{ab}$ & $22,1 \mathrm{~b}$ & $* 27,0 \mathrm{a}$ & $26,2 \mathrm{bc}$ \\
\hline Test. inf. & ${ }^{\mathrm{n}} 7,4 \mathrm{bc}$ & $7,4 \mathrm{~d}$ & $* 1028,0 \mathrm{~cd}$ & $784,5 \mathrm{def}$ & $* 7,3 \mathrm{c}$ & $3,5 \mathrm{e}$ & $* 16,7 \mathrm{de}$ & $19,0 \mathrm{~d}$ & $* 23,9$ de & $22,5 \mathrm{f}$ \\
\hline $\mathrm{CV}(\%)$ & 3,78 & 3,78 & 3,05 & 3,05 & 6,14 & 6,14 & 2,26 & 2,26 & 1,80 & 1,80 \\
\hline
\end{tabular}

${ }^{1}$ Médias seguidas de letras minúsculas distintas, na coluna, diferem entre si pelo teste de Tukey $(\mathrm{p} \leq 0,05)$ e por *, na linha, entre os genótipos de cevada para cada variável resposta, diferem entre si pelo teste $t(\mathrm{p} \leq 0,05)$.

Ao se comparar os genótipos de cevada entre si para cada tratamento observou-se que o MN610 foi superior ao Crioula, ou seja, apresentou menor número de grãos estéreis ao se aplicar iodosulfuron e cyhalofop-p-butyl (Tabela 5). Nos demais tratamentos o genótipo MN610 ou apresentou os maiores números de grãos estéreis ou não ocorreram diferenças significativas.

O número de grãos cheios por espiga foi maior ao se usar o imazethapyr + imazapic, cyhalofop-p-butyl e pyroxsulam, sendo significativamente semelhantes a testemunha capinada, para o genótipo MN610 (Tabela 5). Em relação ao genótipo Crioula observou-se que o uso de cyhalofop-p-butyl propiciou o maior número de grãos cheios por espiga. Já o menor número de grãos cheios foi verificado ao se usar o clomazone, metsulfuron-methyl, 2,4$\mathrm{D}$, penoxsulam e a testemunha infestada. Vargas e Roman (2005) ao aplicarem herbicidas em cultivares de trigo, triticale, centeio e cevada observaram os maiores efeitos fitotóxicos do metsulfuron-methyl sobre a cevada, fato esse também constatado no presente experimento com redução no número de grãos cheios e total por espiga.
Conforme verificado para o número de grãos estéreis observou-se também para o número de grãos cheios que o genótipo MN610 apresentou os melhores resultados para os tratamentos imazethapyr + imazapic, 2,4-D e a testemunha capinada (Tabela 5).

Com relação ao número total de grãos por espiga (Tabela 5) verificou-se resultados distintos da aplicação dos herbicidas, em relação aos genótipos de cevada. O genótipo MN610 apresentou os melhores resultados para o uso de clomazone e de cyhalofop-p-buthyl, os quais não diferiram da testemunha capina. Ainda em relação ao MN610 observou-se os piores resultados ao se aplicar o metsulfuron-methyl.

No genótipo Crioula, o maior número total de grãos foi verificado quando se aplicou o iodosulfuron e o cyhalofop-p-buthyl, sendo 8,0 e $8,8 \%$ superior a testemunha capina, respectivamente (Tabela 5). Para esse mesmo genótipo o pior resultado foi verificado com a aplicação de 2,4-D. Chao et al. (1996) verificaram que o 2,4-D amina reduziu o perfilhamento da cevada, devido ao aumento da dominância apical. Nesse caso os autores observaram que o 2,4-D promoveu aumento no número de grãos por panículas, sendo atribuído 
tal resultado a interferência desse produto sobre a diferenciação floral da cultura.

Os herbicidas imazethapyr + imazapic, clomazone, oxyfluorfen, 2,4-D, penoxsulam e a testemunha capinada demonstraram ser os melhores tratamentos quando aplicados sobre o genótipo MN610 ao se comparar com o Crioula para o número total de grãos (Tabela 5). Ao se comparar os genótipos entre si dentro de cada herbicida, observou-se que o Crioula apresentou o maior número total de grãos por espiga, ao se aplicar o iodosulfuron, clomazone, propanil, metsulfuron-methyl, oxyfluorfen, cyhalofop-pbuthyl e pyroxsulam.

O peso de mil grãos, componente de importância para a qualidade dos grãos de cevada, sofreu variação de acordo com o genótipo em estudo e com o tratamento aplicado (Tabela 6). Para o genótipo MN610, os melhores tratamentos foram observados para propanil, as testemunhas capinada e infestada, iodosulfuron, 2,4-D e cyhalofop-p-buthyl. Os demais tratamentos demonstraram os menores pesos de mil grãos de cevada para o genótipo MN610. Já para o genótipo Crioula a aplicação de 2,4-D demonstrou o maior peso de mil grãos, sendo 9\% superior a testemunha capinada. Santos et al. (2000) ao trabalharem com propanil, fenoxaprop-ethyl, fenoxaprop-p-ethyl, oxadiazon e as misturas compostas de propanil + molinate e propanil $+2,4-\mathrm{D}$, no controle das plantas daninhas, verificaram que o peso de mil grãos de arroz não foi influenciado pelos herbicidas aplicados, nem pela infestação das plantas daninhas.

A produtividade de grãos dos genótipos de cevada foi influenciada de forma diferenciada ao se aplicar os herbicidas para o controle de azevém (Tabela 6). Ao se comparar os tratamentos que levaram aplicação de herbicidas com a testemunha capinada verificou-se redução da produtividade de grãos para o genótipo de cevada MN610, independentemente do produto avaliado. Entre os herbicidas testados o propanil e o 2,4-D foram os que apresentaram as maiores produtividades de grãos, diferindo estatisticamente de todos os demais para o genótipo MN610. Para o genótipo Crioula, os melhores resultados foram observados ao se usar o iodosulfuron e o propanil, sendo que os dois apresentam maiores produtividades de grãos que a testemunha capinada. Constataramse as maiores reduções de produtividades de grãos, 84 e $67 \%$ respectivamente, para os genótipos MN610 e Crioula ao serem tratados com imazethapyr + imazapic. Petter et al. (2011) também observaram redução da produtividade de grãos ao avaliarem a aplicação de diferentes herbicidas para o manejo de plantas daninhas infestantes do arroz.

Ao se comparar os dois genótipos entre si, observou-se que o Crioula apresentou maior produtividade de grãos ao se aplicar iodosulfuron, imazethapyr + imazapic, propanil e pyroxsulam (Tabela 6). O genótipo MN610 demonstrou ser mais produtivo que o Crioula ao se usar o oxyfluorfen, 2,4-D, penoxsulam, as testemunhas capinada e infestada. A perda da produtividade pode estar associada as reduções dos componentes de rendimento das culturas, como número de espigas, quantidades de grãos cheios, massa de mil grãos, conforme observado nas Tabelas 5 e 6 . Ao ser avaliado a fitotoxicidade do herbicida imazethapyr, aplicado em diferentes doses, Hartwig et al. (2008) observaram decréscimo significativo da produção de massa seca nos genótipos de diferentes cultivares de aveia ICA 5, ICA 7, BRS 177, IPR 110, BRS 208 e CD 111. Esse resultado é similar ao observado no presente trabalho, ou seja, as cultivares de cevada respondem de modo diferenciado a aplicação de herbicidas. Esse fato deve-se as características genéticas diferenciadas que as cultivares apresentam, conforme já comentando anteriormente.

Quando os genótipos de cevada MN610 e Crioula foram mantidos livres da competição de azevém apresentaram produtividades de grãos de 112,77 e 127,48\%, respectivamente, maior que a testemunha infestada (Tabela 6). 
Ressalta-se que o genótipo Crioula, consegue se adaptar mais facilmente as condições adversas do meio, no caso do presente trabalho a aplicação de herbicidas, em função de que essa apresenta características mais rusticas se comparado as cultivares modernas de cevada onde os programas de melhorando retiraram determinados genes que lhes conferiam resistência a condições de estresses. Carpentieri-Pípolo et al. (2010) ao trabalharem com variedades crioulas de milho (P-13, P-15 e $\mathrm{P}-05)$ em vários locais, demonstraram potencial produtivo elevado em sistemas de cultivos adversos à cultura.

Tabela 6. Massa de mil grãos (g) e produtividade de grãos $\left(\mathrm{kg} \mathrm{ha}^{-1}\right)$ dos genótipos de cevada em função da aplicação de herbicidas. UFFS, Erechim/RS, 2012/13.

\begin{tabular}{|c|c|c|c|c|}
\hline \multirow{2}{*}{ Tratamentos } & \multicolumn{2}{|c|}{ Massa de mil grãos (g) } & \multicolumn{2}{|c|}{ Produtividade $\left(\mathrm{kg} \mathrm{ha}^{-1}\right)$} \\
\hline & MN610 & Crioula & MN610 & Crioula \\
\hline Iodosulfuron & ${ }^{n s} 41,4 b$ & $41,5 \mathrm{bc}$ & $* 991,1 \mathrm{~d}^{1}$ & $1533,5 \mathrm{a}$ \\
\hline Imazethapyr + imazapic & $* 40,7 \mathrm{bc}$ & $39,7 \mathrm{de}$ & $* 285,6 \mathrm{~h}$ & 389,81 \\
\hline Clomazone & ns $39,9 \mathrm{c}$ & $40,4 \mathrm{~cd}$ & ${ }^{\mathrm{ns}} 640,5 \mathrm{fg}$ & $632,3 \mathrm{~g}$ \\
\hline Propanil & $* 43,6$ a & $39,1 \mathrm{e}$ & $* 1249,1 \mathrm{c}$ & $1354,8 \mathrm{~b}$ \\
\hline Metsulfuron-methyl & $* 40,5 \mathrm{bc}$ & $39,8 \mathrm{de}$ & ${ }^{\mathrm{ns}} 696,5 \mathrm{f}$ & $658,1 \mathrm{fg}$ \\
\hline Oxyfluorfen & $* 40,5 \mathrm{bc}$ & $42,0 \mathrm{~b}$ & $* 1014,8 \mathrm{~d}$ & $961,9 \mathrm{~d}$ \\
\hline $2,4-\mathrm{D}$ & $* 41,2 \mathrm{~b}$ & $44,4 \mathrm{a}$ & $* 1635,8 \mathrm{~b}$ & 863,8 e \\
\hline Cyhalofop-p-buthyl & $* 41,3 \mathrm{~b}$ & $39,8 \mathrm{de}$ & $* 607,1 \mathrm{~g}$ & $527,2 \mathrm{~h}$ \\
\hline Penoxsulam & $* 40,4$ bc & $41,3 \mathrm{bc}$ & $* 868,7 \mathrm{e}$ & $716,9 \mathrm{f}$ \\
\hline Pyroxsulam & $* 39,6 \mathrm{c}$ & $41,4 \mathrm{bc}$ & $* 862,8 \mathrm{e}$ & $1019,9 \mathrm{~d}$ \\
\hline Testemunha capinada & $* 42,9 \mathrm{a}$ & $40,6 \mathrm{~cd}$ & $* 1754,1 \mathrm{a}$ & $1166,3 \mathrm{c}$ \\
\hline Testemunha infestada & $* 43,4 \mathrm{a}$ & 39,9 de & $* 824,4 \mathrm{e}$ & $512,7 \mathrm{~h}$ \\
\hline $\mathrm{CV}$ & 1,18 & 1,18 & 3,12 & 3,12 \\
\hline
\end{tabular}

Ao se analisar a influência dos herbicidas sobre todas as variáveis testadas denota-se que os efeitos dos mesmos ocorrem de maneira diferenciada para o genótipo em que foram aplicados e também de acordo com o produto utilizado. Isso decorre em função das características distintas que os genótipos de cevada apresentam e também das características físico-químicas de cada herbicida que irá conferir maior ou menor tolerância da cultura à estresses ocasionados por agentes xenobióticos.

\section{Conclusões}

O imazethapyr + imazapic apresenta a maior redução nos componentes de rendimento de grãos dos genótipos de cevada MN610 e Crioula. Os herbicidas registrados para o controle de plantas daninhas infestantes da cultura da cevada, o 2,4-D, para o genótipo MN610 e metsulfuron-methyl, para o genótipo Crioula, não apresentaram redução significativa na produtividade de grãos da cevada.
De modo geral o iodosulfuron demonstra a melhor resposta à cultura da cevada, por apresentar o melhor controle do azevém e menor fitotoxicidade aos dois genótipos testados, além do maior índice de produtividade de grãos para o genótipo Crioula.

O herbicida pyroxsulam apresenta ótimo controle do azevém, porém fitotoxicidade elevada e produtividade de grãos intermediária entre os melhores e os piores tratamentos, merecendo assim maiores estudos para fins de sua aplicação na cultura da cevada. As respostas dos genótipos, quanto à aplicação dos herbicidas, foram diferenciadas, para todas as variáveis avaliadas, logo mais trabalhos devem ser conduzidos para testar a eficácia dos herbicidas no controle de plantas daninhas e a fitotoxicidade à cevada.

\section{Agradecimentos}

Os autores agradecem ao $\mathrm{CNPq}$ e FAPERGS pela concessão de auxilio financeiro a pesquisa (processos n.: 482144/2012-2/CNPq 
e 12/2265-3/FAPERGS) e pelas bolsas de produtividade em pesquisa e de iniciação científica.

\section{Referências}

AGOSTINETTO, D. et al. Respostas de cultivares de soja transgênica e controle de plantas daninhas em função de épocas de aplicação e formulações de glyphosate. Planta Daninha, v.27, n.4, p.739-746, 2009.

BALBINOT JR., A. A. et al. Competitividade de cultivares de arroz irrigado com cultivar simuladora de arroz-vermelho. Pesquisa Agropecuária Brasileira, v. 38, n.1, p. 53-59, 2003.

BRASIL. Ministério da Agricultura e Pecuária. AGROFIT. Disponível em: <http://www.agricultura.gov.br>. Acesso em: 05/06/2015.

CARPENTIERI-PÍPOLO, V. et al. Avaliação de cultivares de milho crioulo em sistema de baixo nível tecnológico. Acta Scientiarum. Agronomy, v. 32, n. 2, p. 229-233, 2010.

CHAO, J. F. et al. Influence of nutrients supply and plant growth regulators on phytoxicity of imazamethabens in wild oat. Journal of Plant Growth Regulation, v.13, n.4, p.195- 201, 1996.

CHRISTOFFOLETI, P. J. et al. Carfentrazoneethyl aplicado em pós-emergência para o controle de Ipomoea spp. e Commelina benghalensis na cultura da cana-de-açúcar. Planta Daninha, v.24, n.1, p.83-90, 2006.

DAL MAGRO, T. et al. Suscetibilidade de cultivares de arroz irrigado (Oryza sativa) à deriva simulada do herbicida imazethapyr + imazapic. Planta Daninha, v.24, n.4, p.751759, 2006.

DAS, A. C. et al. Effect of the herbicides oxadiazon and oxyfluorfen on phosphates solubilizing microorganisms and their persistence in rice fields. Chemosphere, v.53, n.5, p.217-221, 2003.
EMBRAPA: Empresa brasileira de pesquisa agropecuária. Centro Nacional de Pesquisa Agropecuária de Solos (Brasília, DF). Sistema brasileiro de classificação de solos. Brasília: Embrapa Produção de Informação; Brasília, DF: Embrapa Solos, 2013. 154p.

FAO - Food and Agriculture Organization of the United Nations. Disponível em: http://www.fao.org/statistics/en/. Acesso em: 02/06/2015.

FENG, P. C.C. et al. Glyphosate inhibits rust diseases in glyphosate-resistant wheat and soybean. National Academy of Sciences, v.102, n.48, p.17290-17295, 2005.

FERREIRA, E. A. et al. Sensibilidade de cultivares de cana-de-açúcar à mistura trifloxysulfuron-sodium + ametryn. Planta Daninha, v.23, n.1, p.93-99, 2005.

GALON, L. et al. Habilidade competitiva de cultivares de cevada convivendo com azevém. Planta Daninha, v.29, n.4, p.771-781, 2011.

GALON, L. et al. Influência de herbicidas na qualidade da matéria-prima de genótipos de cana-de-açúcar. Planta Daninha, v.27, n.3, p. 555-562, 2009.

GALON, L. et al. Manejo de plantas daninhas em cereais de inverno. In: SANTOS, H.P. dos; FONTANELI, R.S.; SPERA, S.T. (Org.). Sistemas de produção para cereais de inverno sob plantio direto no Sul do Brasil. Passo Fundo: Embrapa, p. 255-316, 2010.

HARTWIG, I. et al. Tolerância de Trigo (Triticum aestivum) e Aveia (Avena sp.) a Herbicidas Inibidores da Enzima Acetolactato Sintase (ALS). Planta Daninha, v.26, n.2, p.361-368, 2008.

MARIANI, F. et al. Controle alternativo de Lolium multiflorum Lam. resistente ao herbicida iodosulfurom-metílico. In: CONGRESSO BRASILEIRO DA CIÊNCIA DAS PLANTAS DANINHAS, 28., 2012. Anais... Campo Grande/MS: SBCPD, 2012. CD-ROM. 
NUNES, A.L. et al. Tolerância de espécies de inverno a herbicidas residuais. Scientia Agrária, v.8, n.4, p.443-448, 2007.

OLIVEIRA, A. R.; FREITAS, S. P.; VIEIRA, H. D. Controle de Commelina benghalensis, C. erecta, Tripogandra diuretica na cultura do café. Planta Daninha, v. 27, n. 4, p. 823-830, 2009.

PAULA, J. M. et al. Manejo de Conyza bonariensis resistente ao herbicida glyphosate. Planta Daninha, v.29, n.1, p.217-227, 2011.

PETTER, F.A.; ZUFFO, A.M; PACHECO, L.P. Seletividade de herbicidas inibidores de als diferentes estádios de desenvolvimento do arroz de terras altas. Pesquisa Agropecuária Tropical, v. 41, n. 3, p. 408-414, 2011.

PINTO, J. J. O. et al. Controle de Capim-Arroz (Echinochloa spp.) em função de métodos de manejo na cultura do arroz irrigado. Planta Daninha, v.26, n.4, p.767-777, 2008.

RIZZARDI, M. A. et al. Ação de herbicidas sobre mecanismos de defesa das plantas aos patógenos. Ciência Rural, v.33, n.5, p.957-965, 2003.

RODRIGUES, O. et al. Efeito da aplicação de herbicida hormonal em diferentes estádios de desenvolvimento de trigo (Triticum aestivum $\mathrm{L}$. Cvs. Embrapa 16 e BR 23). Revista Brasileira de Herbicidas, v.5, n.1, p.19-29, 2006.

ROLAS - Rede oficial de laboratórios de análise de solo e de tecido vegetal. Manual de adubação e calagem para os estados do Rio Grande do Sul e Santa Catarina. 10.ed., Porto Alegre: Sociedade Brasileira de Ciência do Solo, 2004, 400p.

ROMAN, E. S. et al. Resistência de azevém (Lolium multiflorum) ao herbicida glyphosate. Planta Daninha, v. 22, n. 2, p. 301-306, 2004.

SANTOS, F. J. et al. Controle químico de plantas daninhas na cultura do arroz irrigado no estado do Ceará. Planta Daninha, v.18, n.1, p.29-37, 2000.
SBCPD - SOCIEDADE BRASILEIRA DA CIÊNCIA DAS PLANTAS DANINHAS. Procedimentos para instalação, avaliação e análise de experimentos com herbicidas. Londrina: 1995.42 p.

TOTTMAN, D. R. The identification of growth stages in winter wheat with reference to the aplication of growth regulator herbicides. Annals of Applied Biology, v.87, n.2, p.213224, 1977.

TUFFI SANTOS, L.D. et al. Glyphosate sobre a resistência à ferrugem (Puccinia psidii) do eucalipto. Planta Daninha, v.25, n.1, p.139147, 2007.

VARGAS, L. et al. Buva (Conyza bonariensis) resistente ao glyphosate na região Sul do Brasil. Planta Daninha, v.25, n.3, p.573-578, 2007.

VARGAS, L. et al. Dose-response curves of Lolium multiflorum biotypes resistant and susceptible to clethodim. Planta Daninha, v.31, n.4, p.887-892, 2013.

VARGAS, L.; ROMAN, E. S. Seletividade e eficiência de herbicidas em cereais de inverno. Revista Brasileira de Herbicidas, v.4, n.3, p.110, 2005. 